\title{
Comparative Studies on Non-Divergent Load Flow Methods in Well, Ill and Unsolvable Condition
}

\author{
Amidaddin Shahriari, Ab. Halim Abu Bakar, Hazlie Mokhlis \\ Department of Electrical Engineering, University of Malaya, 50603 Kuala Lumpur, Malaysia
}

\begin{abstract}
This paper describes a critical evaluation of NonDivergent Load flow methods in well, ill and unsolvable conditioned systems. The comparison studies deals Multiple Load Flow Solution (MLFS) based Second-Order Load-Flow (SOLF) in polar coordinate and Continuation Load Flow (CLF). The analytical bases, ability consideration of theses methods to return operation of power system from unsolvable to solvable region solution. Attention is given to the problems and techniques of to provide optimal recommendations of the parameters, that using in these Non-Divergent Load flow methods to lead to solvable region, based on inequality constraints of power system. A part of the survey, this paper also presents the comparison of numerical result using different type of aforesaid load flow methods for well and ill- conditioned systems. Accordingly, load flow simulation has been solved using the $\mathrm{C}++$ programming.
\end{abstract}

Index Terms-- Second-Order Load-Flow, Multiple load flow solution, Continuation Load Flow, ill-conditioned system, unsolvable condition.

\section{INTROCTDUION}

A $\mathrm{t}$ first of 70's, ability of load flow methods were considered to over come, outage security assessment, optimization and stability [1],[31]. Thus, the load-flow studies required the less computer memory and far less execution time. These issues were addressed in the early 1970 's when Stott and Alsac presented Fast Decoupled Load Flow (FDLF) model [1]. The FDLF was the usual choice in transmission applications. But, during 70's power system operation was confronted to ill conditioning. The ill conditioning for FDLF was high ratios of lines $r / x$, connections of very low and very high impedance lines at a bus that could cause FDLF to converge slowly or was divergent [2]. However, when reliability and accuracy, rather than speed of response, was a concern, or when the decoupling principle did not hold, the Newton-Raphson (NR) was the preferred .But conventional NR was poor convergence for radial distribution systems. There were heavy loading at some buses and results in low voltages at these [12], [36]. On other meaning that as the system loading approaches critical loading the Jacobian matrix tends to become singular[3], [39]. Under this condition, even the NR was encountered difficulties to reach a solution. Because, efficient sparsity oriented implementation of NR decreases and no solution from initial estimate increases [7],[17]. This issue has motivated the development of alternative methodologies, based on the NR iterative scheme, specifically [34].

At end of 70's, second-order load-flow (SOLF) methods began to appear [5], [20]. First second order load f low technique based on the Taylor series expansion of a load flow equation was in polar coordinate form. In many cases, this second order required lesser iterations, had better convergence characteristics in almost all the load flows attempted by the authors than NR technique. Moreover, it had also been shown that the elements of the second order coefficient matrix need not be stored separately. Rectangular forms of second order method as a fast load flow method retaining nonlinearly (Iwamoto's Method) was introduced in 1978 [5], [25] .Its specific character was a fixed or constant matrix was used throughout the iteration process [33]. Regarding this point, was claimed that Iwamoto Method was used for training simulators in Japan [18], for its fastness. Increasing demand without expansion of transmission facilities will lead transmission and distribution systems operate very close to critical loading condition (voltage collapse) [32],[37],[38]. Under this condition, conventional load flow Newton methods never converges. Divergence can also occur when poor initial estimation are used to begin the solution process [22], [23]. Thus, it is important that a computationally efficient technique be developed to both quantify the degree of unsolvability, and to provide optimal recommendations of the parameters to change and returning to a solvable solution [19], [24],[28]. Therefore, at beginning of 80's, Multiple Load Flow Solution (MLFS) methods were presented [6], [7], [31]. As predictor, check best slope from a critical point i.e. from critical initial value to safe margin zone of voltage stability, in every step to become converge with out changing in control variables that is shown for a $\mathrm{P}-\mathrm{V}$ or $\mathrm{Q}-\mathrm{V}$ of typical bus as voltage-load curve in Fig1. Among theses methods, Iwamoto and Tamura [6] presented the robust non-divergent load flow methods in well and ill conditioned based on the SOLF Iwamoto method [5].

After that concept Power System Security (PSS) under highly stress contingency situation in bulk power system penetrated in power system studies during 80's [8], [26]. At end of 80's, the Continuation Load Flow (CLF) was introduced at 90's first [14]. As the simple definition, according to Fig.1, to modify gradient curve of MLFS methods by active and reactive power injecting of system resources and compensating is CLF as corrector [15]. In method, parameterized load flow equations 
are solved and the parameter provides an indication whether the system has or has not a solution [21]. On other meaning, maximum loading condition of a system as a saddle-node bifurcation is defined by CLF [11], [13],[16]. Furthermore, the typical fig 1 shows the unsolvable case, the power flow solution does not exist. In this case power systems is become more heavily loaded, then, increase the number of situations where the power flow equations have no real solution [13], [27]. These methods provide optimal recommendations of the dependant and independent variable of power system to return respectively from ill-conditioned to feasible region and unsolvable solution to infeasible region [9],[11], [29].

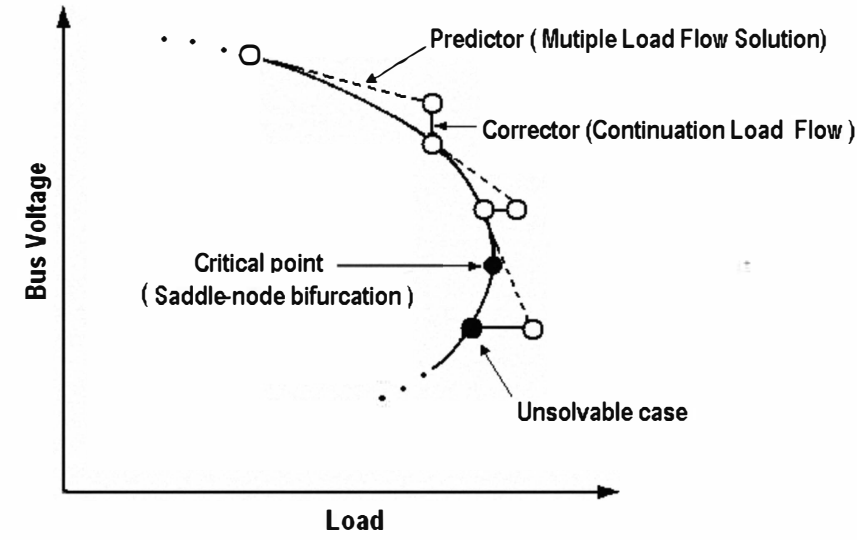

Fig. 1. Sequence of calculation in a Multiple Load Flow Solution and Continuation Load Flow method.

This paper aim to develop Comparative Studies on NonDivergent Load Flow Methods in Well, Ill and Unsolvable Condition to deal with the MLFS method based on the SOLF in polar coordinate [6],[10],[20] and the CLF method for well, ill and unsolvable - conditioned cases respectively [13], [14], [15], [16].firstly, the SOLF analytic geometry indicates that has better performance in convergence time execution and mismatch error in comparison with the NR in well and ill conditioned system. In following appears that SOLF in polar coordinate form performs robust in non-divergent load flow method rather than the SOLF in rectangular coordinate. In order to the SOLF, MLFS based on optimal multiplier is evaluated in ill condition and is shown indeed CLF is the modified MLFS in confronting stressed or unsolvable systems. Numerical result that has been carried out for 13 bus illconditioned [7] and standard IEEE 30 bus system, confirms the survey of this paper. In addition, load flow simulation has been solved using the $\mathrm{C}++$ programming [40].

\section{FIRST AND SECOND ORDER TERM THEORY IN NEWTON RAPHSON LOAD FLOW METHOD}

The power flow problem of electrical power can be written as a set of nonlinear equations, the following form:

$$
F(X, Y)=0
$$

Where X - vector of uncontrolled ( dependent) variables;
Y - vector of controlled ( independent) variables;

F- vector function of load flow

For solving (1) is needed to use a numerical iterative technique. The $i$ th iteration of classical the (NR) algorithm based as the first - order Taylor series expansion of $F(X a, X b)$ for two variables i.e., voltage amplitudes and phases at buses, is as follow

$$
\begin{aligned}
& F\left(X a_{i}+\Delta X a_{i}, X b_{i}+\Delta X b_{i}\right)-F\left(X a_{i}, X b_{i}\right) \\
& -\left\lfloor\Delta_{X a, X b} F\right\rfloor_{i}\left[\Delta X a_{i}, \Delta X b_{i}\right] \approx 0
\end{aligned}
$$

Newton's method is very reliable and extremely fast in convergence in well conditioning. In this condition, the power flow solution exists and is reachable using a flat initial guess (e.g., all load voltage magnitudes equal to 1 and all bus voltage angles equal to 0 ). This case is the most common situation. Thus, numerical newton method can approach to optimum point. By starting from an initial guess $\left(X a_{0}, X b_{0}\right)$ the series converges towards solution point in last iteration. The algorithm stops if the variable increments are lower than a given tolerance or the number of iterations is greater than a given limit.

Because of neglecting the high order terms, (2) is an approximate equal of (1). The most important fact in (1) is that only the second derivative exist and no terms above third. Actually, (1) is a quadratic function respect to depend variable [11], [13], [22]. Therefore, Second Newton -Raphson Method (SNRM) as exact expression is formulated as follows :

$$
\begin{aligned}
& F\left(X a_{\imath}+\Delta X a_{i}, X b_{\imath}+\Delta X b_{i}\right)-F\left(X a_{i}, X b_{l}\right)-\left[\Delta_{X a, X b} F\right]_{i}\left[\Delta X a_{i}, \Delta X b_{i}\right] \\
& -\frac{1}{2}\left[\Delta X a_{i}, \Delta X b_{i}\right]^{T}\left[\Delta^{2}{ }_{X a, X b} F\right]_{[}\left[\Delta X a_{i}, \Delta X b_{i}\right]=0
\end{aligned}
$$

The paramount distinction of SNRM to Newton method is apparent in (1). Since, (1) is quadric function respect to $[\triangle X a, \Delta X b]$. Therefore, a pair of the correction value indeed exists at each iteration that are given

$$
\begin{aligned}
& X_{1}=\Delta X_{1}+X_{0} \\
& X_{1}^{\prime}=\Delta X_{2}+X_{0}
\end{aligned}
$$

Likewise, for more comprehension Fig.2 depicts the sample model of geometry performance of the assumed a pair of $\Delta X$ in term of $F(X)$ for SNRM and NR methods. In order Fig.2, the speedup of taking a step towards optimum point ( maxim and minimum) $F\left(X_{S 0}\right)=S_{0}$ in SNRM can be faster converge than NR method in well and ill- conditioned system. Second distinction shows itself in ill conditioned. The ill conditioning has a prevailing radial topology (weaklyinterconnected) and atypical transmission lines parameters (high ratios of lines $r / x$, connections). .Furthermore, its generation and load busses are not satisfied. According to 
these conditions, on the one hand power system operates very close to critical loading (voltage collapse point).

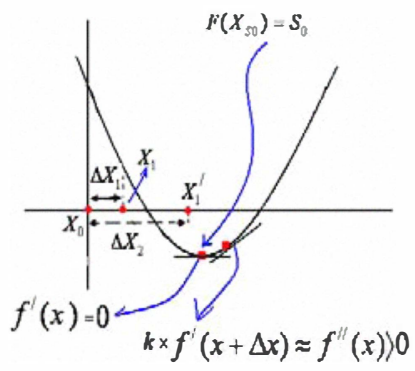

a) SOLF method



b) NR method
Fig.2. Comparison of $\Delta X$ and performance of the SNRM to approach optimum point.

On other hand, determinant of jacobain matrix is zero, because of singular jacobain matrix. Nevertheless, this zero does not mean system approach optimum or stable point (voltage stability). On the contrary leads to unstable point saddle point. For detecting saddle point, the second derivative matrix of SNRM executes as Hessian matrix.

Let us to define the second derivative of $F(X)$ respect to $X$ in optimum points in Fig.2 as

$$
\begin{aligned}
& F^{\prime \prime}(X)=\lim \frac{F^{\prime}(X+\Delta X)-F^{\prime}(X)}{\Delta X} \\
& =\lim \frac{F^{\prime}(X+\Delta X)-0}{\Delta X} \approx \frac{1}{\Delta X} F^{\prime}(X+\Delta X) \\
& \approx k \times F^{\prime}(X+\Delta X)
\end{aligned}
$$

Since, if the (6) has positive sign $X$ then $F(X)$ accedes a local minimum at $X$. In negative sign of $(6), F(X)$ accedes a local maximum at $X$. The performance of this issue in load flow is correspond to the Hessian matrix. Respectively ,The negative and positive definite of the Hessian matrix at $\mathrm{Xa}, \mathrm{Xb}$ leads (1) to local maximum and minimum [19],[25]. Furthermore, If the Hessian has both positive and negative eigenvalues then $(X a, X b)$ is a saddle point for (1) [16]. Otherwise the Hessian test is inconclusive.

The particular difficulty of SNRM is to calculate of the correction value at each iteration. The quadric matrix of $\left\lfloor\Delta X a_{i}{ }^{2}, \Delta X b_{i}{ }^{2}\right\rfloor$ can not be solved in straightforward manner as same in NR method. For obtaining $\left[\Delta X a_{i}, \Delta X b_{i}\right]$ a solution of without excessive computer attempt, the first SNRM's in polar and rectangular coordinate used Gauss-Seidel methodology [5][20]. Accordingly, (3) is modified as follows :

$$
\begin{aligned}
& F\left(X a_{i}+\Delta X a_{i}, X b_{i}+\Delta X b_{i}\right)-F\left(X a_{i}, X b_{i}\right) \\
& -\frac{1}{2}\left[\Delta X a_{i}, \Delta X b_{i}\right]^{T}\left[\Delta^{2}{ }_{X a, X b} F\right]\left[\Delta X a_{i}, \Delta X b_{i}\right]=\left[\Delta_{X a, X b} F\right]_{i}\left[\Delta X a_{i}, \Delta X b_{i}\right]
\end{aligned}
$$

Indeed, $\left[\Delta X a_{i}, \Delta X b_{i}\right]$ at iteration $\mathrm{i}$ determined by $\left[\Delta X a_{i-1}, \Delta X b_{i-1}\right]^{T}\left[\Delta X a_{i-1}, \Delta X b_{i-1}\right]$ in straightforward manner and prior iteration. Other meaning is that a internal loop relevant to calculating jacobian matrix $\left\langle\Delta_{X a, X b} F\right]_{i}$ is added to conventional NR load flow. The authors [6] presented rectangular coordinate of SNRM based on the fixed Jacobian method (FJM). The defect of this method appeared in reliability studies while configuration of jacobian matrix is modified frequently. Since, under this condition each of loadflow solution represents a different system in term of its topology and/or status of its regulated buses. As shown in [15], polar coordinate form of the SNRM [20] provide faster and less requiring storage solution. In addition, the SNRM based on polar formulation performs more reliable that is particularly apparent in the highly stressed system [15], [18]. The set of second terms equations for power mismatch in the SNRM polar form contains twenty elements that each the active or reactive power mismatch include ten elements. The numerous number of these elements drives calculation of second term matrix very complicate. This paper follows and modifies the SNRM in [20] that neglects the some of these elements that uses in Fast Decoupled Load Flow (FDLF) model. how and how many, elements of second term matrix using depends on system configurations is not mature much more research is necessary.

\section{EVALUATION OF LOAD FLOW QUADRATIC FUNCTION}

Let us to suppose load flow equation (1) for a bus system $\mathrm{k}$ in the $i$ th iteration as

$$
F k_{i}\left(X a k_{i}, X b k_{k}, Y a k_{k}, Y b k_{k}\right)=0
$$

Respectively $(X a, X b, Y a, Y b) \in R^{n}$ correspond to real and imaginary of voltage bus amplitude and injected bus active and reactive power. A quadratic function for bus $\mathrm{k}$ is given by [11], [13], [22]:

$$
\alpha X^{2}+\beta X+\gamma=0
$$

To evaluate of (9) roots, presents a pair of bus k voltage at every iteration. For mathematical power system comprehensive analyzing of bus $\mathrm{k}$, assumed descriptive geometry of (9) for two solutions. From observation of power system control steady state operation in power flow, power system operate as same as fixed point theory. It means the trajectory of studied bus voltage (X) is fixed in optimum or concave point [19], [28]. For simplification of bus $k$ description geometry, convex form is supposed in instead of concave form [34], [39].

According to this hypothesis, solid geometry of (9) is illustrated in Fig. Suppose, we start a load flow an initial guess or first operating point $F\left(X a_{C}, X b_{C}\right)=C$ in Fig.3, using the SNRM and NR in polar coordinates. After several iterations, two solutions $\mathrm{A}$ and $\mathrm{B}$ should be located proximate vector $\left[\Delta X_{1}\right]$. Exact and optimum solution of bus $\mathrm{k}$ is considered $\mathrm{B}$. the principal issue is that how can divert to solution $\mathrm{B}$, while load flow calculation is converging to 
solution $\mathrm{A}$. If solution $\mathrm{B}$ exists in extension line vector $\left[\Delta X_{i}\right]$ that crosses point $\mathrm{A}$, by using the SNRM can approach to solution $\mathrm{B}$ that is shown as $\mathrm{AB}$ segment line in Fig.4. Fig.4 is correspond to the contour form of Fig.3. the

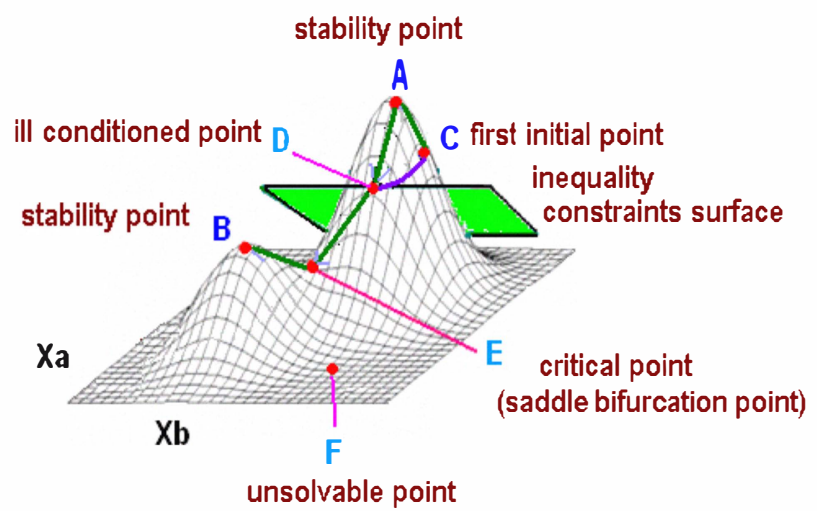

Fig .3. The supposed solid geometry of (8) respect to $(X, Y)$

This is because, the Newton method performance is sensitive to the behaviors of the load flow functions and hence to their formulation. The more linear they are, the more rapidly and reliably Newton's method converges.

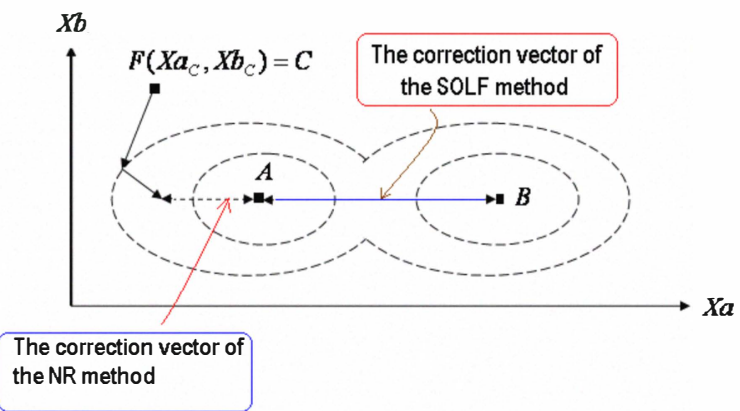

Fig.4. The contour form of supposed solid geometry of $(8)$ respect to $(X, Y)$.

On the Other hand, non smoothness, i.e., humps, in any functions of (8) in the region of interest can cause convergence delays, total failure, or misdirection to a non useful solution. The variation of load flow function is correspondent to changing of power system topology from voltage and system frequency stability (well) to instability conditioned such as voltage collapse. First step of this procedure is nominated ill condition. Typically, this situation is due to the fact that the region of attraction of the power flow solution is narrow or far away from the initial guess. But, the load flow equations have real solution. The ill conditioning is occurred by adding some equality and inequality constraints as variables and functions to load flow equations that should be satisfied coincidentally. Therefore, the set of nonlinear equality and inequality constraints can be given [28]:

$$
\begin{aligned}
& G(X, Y, U) \leq 0 \\
& W(X, Y, U)=0
\end{aligned}
$$

$U$ is control the vector of control variable s that includes Qlimit violation, generate outage, newly-turned on generator and so on. Fig.3 depicts a supposed typically system constraint as approximately flat surface. The geometric concept of supped surface performance is to decline the purpose solution point $\mathrm{A}$ to point $\mathrm{D}$.moreover, operating power system close to its security margins that occur in heavy loaded in planning application and contingency analysis leads system to unsolvable cases. This operation is that By increasing load demand, point $\mathrm{A}$, forced to locate in the boundary region, accordingly by becoming more highly stress is dropped in unsolvable region that are supposed points $\mathrm{E}$ and $\mathrm{E}$ in Fig.3 respectively. Nevertheless, the number of situations that the load flow equations have no real solution, increases. As was mentioned in previous section, to approach solution $\mathrm{B}$ is accomplished under concept of Multiple and Continuation load flow Load model, as, the optimal direction, to move in dependent and independent variable space to return to power flow solvability zone [13], [14], [17].

\section{MULTIPLE LOAD FLOW SOLUTION METHOD}

At beginning of 80's, Multiple Load Flow Solution (MLFS) methods was presented, as predictor, check best slope from a critical point from critical initial value to safe margin zone of voltage stability at each step to be convergence with out changing in control (independent) variables [6], [7], [30] . On other meaning the predictor is to adjust the size of vector $\left[\Delta X_{i}\right]$ and specifying optimal value to takes a step towards the best stability solution in ill conditioned system, i.e. solution $B$ in Fig.2 and Fig.3. Hence, the modification of step update is formulated as follows:

$$
X_{i+1}=X_{i}+\lambda \Delta X_{i}
$$

Rewriting (3) with the scalar multiplier gives :

$$
\begin{aligned}
& \left.F\left(X a_{i}+\Delta X a_{i}, X b_{i}+\Delta X b_{i}\right)-F\left(X a_{i}, X b_{i}\right)-\lambda_{i} \mid \Delta_{X a, X b} F\right]_{i}\left[\Delta X a_{i}, \Delta X b_{i}\right] \\
& -\lambda_{i}^{2} \frac{1}{2}\left[\Delta X a_{i}, \Delta X b_{i}\right]^{T}\left[\Delta^{2}{ }_{X a, X b} F\right]\left[\Delta X a_{i}, \Delta X b_{i}\right]=0
\end{aligned}
$$

By plotting scalar cubic of (13) as objective function (L) that is given in (14), respect to $\lambda$ is shown the practically a pair concave steady state point ( local minimum) and a saddle point ( local maximum) that respectively correspond to A, B and $\mathrm{E}$ in Fig.3.

$$
\begin{aligned}
& L=\mid F\left(X_{i}+\Delta X_{i}, Y_{i}+\Delta Y_{i}\right)-F\left(X_{i}, Y_{i}\right)-\lambda_{i}\left[\Delta_{X, Y} F\right]_{i}\left[\Delta X_{i}, \Delta Y_{i}\right] \\
& -\lambda_{i}{ }^{2} \frac{1}{2}\left[\Delta X_{i}, \Delta Y_{i}\right]^{T}\left[\Delta^{2}{ }_{X, Y} F\right]\left[\Delta X_{i},\left.\Delta Y_{i}\right|^{2}\right.
\end{aligned}
$$

If system has a pair of near solution, then according to Fig.5, the degree of polynomial of (14) differentiation respect to $\lambda$ becomes three. In this situation three real roots, are exist for 
$\frac{\partial L}{\partial \lambda}$. In ascending order to roots, suppose $\lambda_{1}, \lambda_{2}$ and $\lambda_{3}$. that are correspond to A, E and B as concave stability solution for $\mathrm{A}$ and $\mathrm{B}$ and bifurcation solution point $\mathrm{E}$ as well as instability solution in real power system.

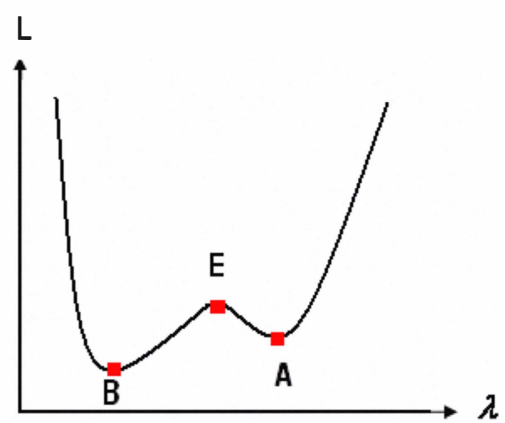

Fig .5. Illustration of scalar cubic of (13) respect $\lambda$

Discussed method is based on the optimal multiplier in the concept of nonlinear programming problem, introduced in and has been developed in [11], [39]. Although, Several methods were presented that deal with MLFS, but most of them the computation of MLFS methods require more analytic effort [9], [10], [12], [21]. Another difficulty for MLFS is apparent in defining maximum loading level that system can supply for unsolvable case. Therefore, Continuation load flow Load (CLF) methods by getting the voltage collapse as it main objective could control level of natural load increasing by active and reactive power compensating.

\section{THE CONCEPT OF CONTINUATION POWER FLOW}

At 90's Continuation load flow Load (CLF) was introduced first. As the simple definition, to modify gradient curve of MLF methods by active and reactive power injecting and compensating is CLF as corrector is shown in Fig.1. Typically, the issue is that the loading level of the network is too high then CLF can define correspond loading level and generator power. A simple method for inserting load parameter in to the is to define as constant power load model [15], [16].

The modified and depended (1) on scalar parameter $\mu$ is given:

$$
K(T, \mu)=0
$$

Where $T \in R$ that $T$ consists $(X, Y)$ and $\mu$ present the multiple load and generator powers

$$
0 \leq \mu \leq \mu_{\text {Critical }}=0
$$

Differentiating (16) at a generic steady state point is as follows :

$$
\frac{\partial K(T, \mu)}{\partial T} d t+\frac{\partial K(T, \mu)}{\partial \mu} d \mu=0
$$

Then, corrector step is given by

$$
\frac{d T}{d \mu}=-\frac{\partial K(T, \mu)}{\partial \mu} \times \frac{\partial K(T, \mu)^{-1}}{\partial T}
$$

By adding the correction value to initial solution, next approximate solution is expressed

$$
K(T+\Delta T, \mu+\Delta \mu)=K(T, \mu)+\frac{d T}{d \mu} \Delta \mu
$$

It is apparent that used optimization method in CLF is based on the decent gradient method [34].

In order to apply a locally parameterized continuation technique to the power flow problem, a load parameter must be inserted into the equations. While there are many ways this could be done, only a simple example using a constant power load model will be considered in this paper. Since, predominance of the CLF for unsolvable cases is obvious in getting the part of the curve (1) or (15) in Fig.3, indeed in practically system in Fig.5. In this sense, it is considered as a the constrain equation of the step size along the length of the got part of Fig.2 as follows :

$$
\left(X_{i}-X_{i}(\text { critical })\right)^{2}+\left(\mu_{i}-\mu_{i}(\text { critical })\right)=\Delta s^{2}
$$

In geometrically concept, to modify path of convergence in point B from C-D-E-B, instead of the path of A-D-E-B.. Furthermore CLF is used to determine peak load demand as boundary region between ill conditioned (infeasible region ) and unsolvable region.

\section{THE COMPARATIVE STUDY TESTES}

In order to approach described the SOLF in polar coordinate and the CLF are tested for IEEE 30 bus test system and 13 ill conditioned system [7] based on $\mathbf{C}++$ programming that has been used for this case study. For well conditioned, the 30bus system is used to examine the effect SOLF in polar coordinate on the convergence time execution and the convergence mismatch. Hence, the SOLF in polar coordinate comparison with the NR method bout max error and CPU time of used PC based second for the IEEE 30 bus system at each iteration is given in Table I and II respectively. It was discussed that after first iteration, the SOLF coordinates in the load flow computation.

TABLE I

THE PERFORMANVE OF THE NR METHOD FOR SOLVING IEEE 30

\begin{tabular}{|c|c|c|c|c|}
\hline Iteration & 1 & 2 & 3 & 4 \\
\hline CPU time(s) & 0.25 & 0.281 & 0.328 & 0.356 \\
\hline Max error & 1.88001 & 0.0120031 & 0.00109039 & $7.94985 \mathrm{e}-09$ \\
\hline
\end{tabular}

Thus, Table I shows that the speedup of computation time at each iteration correspond to its CPU time, compare with NR in Table II are less. Hence, is shown to be fast of SOLF the speedup in taking a step towards optimum point. In order to the accuracy of the SOLF mismatch vector at each iteration 
ratio to The NR is demonstrated the SOLF as exact load flow model. Although, respectively in this test system number of iteration necessary obtain solution for the SOLF and NR are 5 and 4. according to SOLF, reusing the Jacobian twice before updating it, gives an overall best performance that used in this test. Furthermore, should be mentioned that the rectangular form of SOLF convergence time execution is 0.321 (s) that has been carried out for 30 - bus system.

TABLE II

THE PERFORMANVE OF THE SOLF METHOD FOR SOLVING IEEE 30

\begin{tabular}{|c|c|l|c|c|c|}
\hline Iteration & 1 & 2 & 3 & 4 & 5 \\
\hline $\begin{array}{c}\text { CPU } \\
\text { time(s) }\end{array}$ & 0.156 & 0.188 & 0.234 & 0.266 & 0.313 \\
\hline $\begin{array}{c}\text { Max } \\
\text { error }\end{array}$ & 1.88001 & 0.012005 & 0.0017524 & 0.00014524 & $2.2072 \mathrm{e}-12$ \\
\hline
\end{tabular}

The shown 13 bus system line diagram in Fig.6 is tested as ill conditioned system. Because of certain radial system type, the heavy buses loading, the position of the slack-generator and the two series capacitors. The characteristic of this system forces jocobian matrix to become singular.

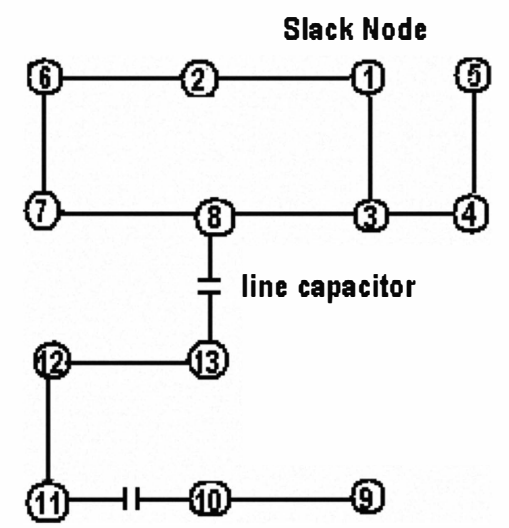

Fig.6. The line diagram of 13 bus ill- conditioned system.

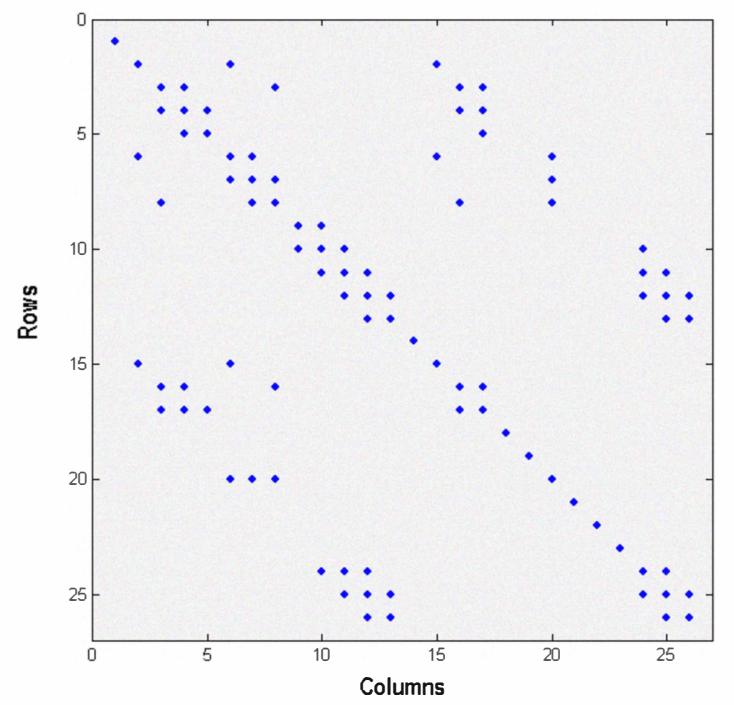

Fig .7. Jacobian matrix for 13 ill conditioned system
Since, eigenvalues of studied jocobian matrix are very sensitive to small changing in its variable state (dependent) variables. Regarding this issue, correspond jocobian matrix absolute value are depicted as conical in diagonal elements of jocobian matrix in Fig. 8 .

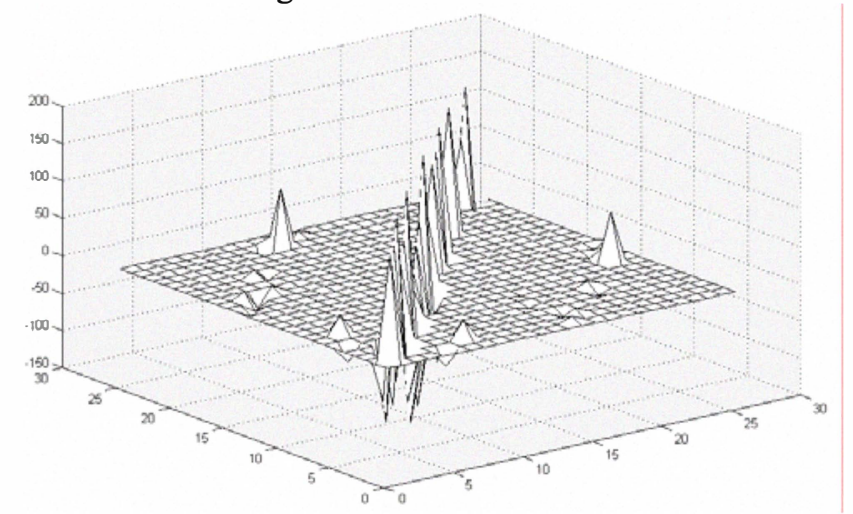

Fig .8. solid geometry of jocobian matrix of 13 bus ill- conditioned system

Under this condition, ration of maximum eigenvalue to minimum eigenvalue as condition the number of the jacobain is very high, in studied ill system is 10000 . This leads to round off error agglomerations during the course of iterative solution and may give rise to oscillations or divergence of power flow solution. First of all, aforementioned system is tested by SOLF and result is given in Table III. This is clear fro table that for converging, approached mismatch value in last iteration is far away from 0.0001 as minimum standard max mismatch error .thus, based newton load flow method such as SOLF and Newton raphson fail to converge.

TABLE III

THE PERFORMANVE OF THE SOLF METHOD FOR SOLVING 13 - BUS ILL CONDITIONED SYSTEM

\begin{tabular}{|c|l|l|l|l|l|}
\hline Iteration & \multicolumn{1}{|c|}{1} & 2 & 3 & 4 & 5 \\
\hline $\begin{array}{c}\mathrm{CPU} \\
\text { time(s) }\end{array}$ & 0.047 & 0.078 & 0.094 & 0.109 & 0.125 \\
\hline Max error & 1.00203 & 4.36341 & 3.00225 & 0.905509 & 0.405434 \\
\hline
\end{tabular}



Fig 9. The performance of CLF in defining critical point of Bus\#13.

For returning 13-bus ill conditioned system to salvable solution, the CLF has been used. Since Continuation power flow defines active and reactive power limitations as load parameter $\mu$ to control buses angles and voltage amplitude 
power line flow by power system resources. Therefore, Bus\#13, in vicinity of heavy load demanding Bus \#12 and series capacitor in line 13-8, is used to illustrate the effect the CLF critical point as voltage instability for Bus\#13. The performance of CLF in defining critical point i.e., $\mathrm{V}=1.21529$ P.U and injected correspond reactive power 1.8 P.U ( base 1000 MVA), in Fig.9, in due to freezes the receding of system operation point in Bus\#13 from ill conditioned case to unsolvable.

\section{CONCLUSIONS}

In this paper, first of all, the performance Multiple Load Flow Solution and Continuation Load Flow as non-divergence load flow methods based on second order load flow in polar coordinate were evaluated, for well, ill conditioned and unsolvable system in mathematical form. In other to this comparative study and confirm it the correspond numerical result was presented that different type of aforesaid load flow methods for well and ill- conditioned systems were used based on $\mathrm{C}++$ programming. The conducted survey in this paper is part of on-going research in power system group at University Of Malaya. to find new robust model of second order load flow for well, ill and unsolvable in practically bulk power system the following these features; (a) improve convergence characteristic (b) reduce computational process of load flow analysis .

\section{REFRENCES}

[1] B. Stott, "Review of load-flow calculation methods," Proc. IEEE, vol62, pp. 916-929, Jul. 1974.

[2] Felix F. Wu," Theoretical Study Of The Convergence Of The Fast Decoupled Load Flow", IEEE Trans. Power Syst., Vol. PAS-96, pp.269 $-275, \mathrm{Jan} / \mathrm{Feb} .1977$.

[3] G. X. Luo and A. Semlyen, "Efficient load flow for large weakly meshed networks," IEEE Trans. Power Syst., vol. 5, no. 4, pp1309-1316, Nov. 1990

[4] B. Stott, "Effective starting process for Newton-Raphson load flows," IEEE Trans. Power App Proc. Inst. Elect. Eng., vol. 118, no. 8, pp. 983987, Nov. 1971

[5] S. Iwamoto and $\mathrm{Y}$. Tamura, "A fast load flow method retaining nonlinearity ," IEEE Trans. Power App. Syst., vol. PAS-97, pp . 15861599, Sep./Oct.1978.

[6] S. Iwamoto and Y. Tamura, "A load flow calculation method for ill conditioned power systems," IEEE Trans. Power App. Syst., vol.PAS100,pp. 1736-1743, Apr. 1981

[7] S. C. Tripathy, G. D. Prasad, O. P. Malik, and G. S. Hope, "Load-flow solutions for ill- conditioned power systems by a Newton-like method," IEEE Trans. Power App. Syst., vol. PAS-101, pp. 3648-3657, Oct. 1982.

[8] M. D. Schaffer and D. J. Tylavsky, "A nondiverging polar form Newtonbased power flow," IEEE Trans. Ind. Appl., vol. 24 870-877, Sep./Oct. 1988

[9] L. M. C. Braz, C. A. Castro, and C. A. F. Murari, "A critical evaluation of step size optimization based load flow methods," IEEE Trans. Power Syst., vol. 15, no. 1, pp. 202-207, Feb. 2000.

[10] J. E. Tate and T. J. Overbye, "A comparison of the optimal multiplier in polar and rectangular coordinates," IEEE Trans. Power Syst., vol. 20 no. 4, pp. 1667-1674, Nov. 2005.

[11] K. Iba, H. Suzuki, M. Egawa, and T.Watanabe, "A method for finding a pair of multiple load flow solutions in bulk power systems," IEEE Trans. Power Syst., vol. 5, no. 2, pp. 582-591, May .1990.

[12] T. J. Overbye and R. P. Klump, "Effective calculation of power system low -voltage solutions," IEEE Trans. Power Syst., vol. 11, no. 1, pp.7582, Feb. 1996.

[13] T. J. Overbye, "A power flow measure for unsolvable cases," IEEE Trans Power Syst., vol. 9, no. 3, pp. 1359-1365, Aug. 1994.
[14] V. Ajjarapu and C. Christy, "The continuation power flow: A tool for steady state voltage stability analysis," IEEE Trans. Power Syst., vol. 7, no. 1, pp. 416-423, Feb. 1992.

[15] - F. Milano, "Continuous Newton's Method for Power Flow Analysis," IEEE Trans. Power Syst, Vol. 24, no. 1, pp. 50-57, February. 2009.

[16] R. J. Avalos, C. A. Cañizares, F. Milano, and A. Conejo, "”"Equivalency of Continuation and Optimization Methods to Determine Saddle-node and Limit-induced Bifurcations in Power Systems," IEEE Trans. Power Cir. Syst, vol. 56, no. 1, pp. 210-223. January. 2009.

[17] A. Z. de Souza, C. A. Cañizares, and V. H. Quintana, "New Techniques to Speed Up Voltage Collapse Computations Using Tangent Vectors," ," IEEE Trans. Power Syst, vol. 12, no, pp, 1380-1387. 3, August .1997.

[18] A. Keyhani, A. Abur, and S. Hao, "Evaluation of power flow techniques for personal computers," IEEE Trans. Power Syst, vol. 4, no, pp 817 826. May .1989.

[19] V.A. Venikov, V.A. Stroev, V.I. Idelchick, and V.ITarasov, "Estimation of electrical power-system steady-state stability," IEEE Trans. Power App.Syst., vol. PAS-94, pp. 1034-1041, May/June .1975.

[20] M. S. Sachdev T. K. P. Medicherla, "A Second Order Load Flow Technique," IEEE Trans. Power App.Syst, Vol. PAS-96, no. 1,pp. 189 197. February .1977.

[21] Shao-Hua Li and Hsiao-Dong Chiang, "Continuation Power Flow With Nonlinear Power Injection Variations: A Piecewise Linear Approximation," IEEE Trans. Power Syst, VOL. 23, NO. 4, Nov. 2008.

[22] R. Wilson Long, "Inherent Constraints Control Convergence A New Method in the Long Look at Load Flow," IEEE Trans. Power App.Syst, Vol. PAS-88,no. 1, Jan .1969

[23] P. R. Bijwe and S. M. Kelapure," Nondivergent fast power flow methods,"IEEE Trans. Power Syst., vol.18, pp.633-638, May. 2003.

[24] Sasson, A.M, "Nonlinear Programming Solutions for LoadFlow,Minimum-Loss, and Economic Dispatching Problems,"IEEE Trans. Power App.Syst, Vol. PAS-88, no.4,pp.399 - 409,April. 1969.

[25] W. Hubbi.,"The mismatch theorem and second-order load-flow algorithms," IEE Proc, Vol. 132, Pt. C, no. 4 pp.189-194, July 1985.

[26] Robert J. Wamser and Ilya W. Slutsker," Power Flow Solution By Newton- Raphson Method In Transient Stability Studies," IEEE Trans. Power App.Syst., Vol. PAS-103, no. 8, pp. 2299-2307, August. 1984.

[27] Rivas. R.Land Ullod. C.L ,"Automatic Blackout Recovery Through a Deterministic Load Flow," in Proc.2006 IEEE Power Engineering Society Transmission and Distribution Conf .pp.1-6.

[28] B. Stott and J.L.Marinho," Linear Programming For Power -System Network Security Applications ", IEEE Trans. Power Syst., Vol .PAS98. pp.837-848. May/June. 1979.

[29] Y. Chen and C. Shen, "A Jacobian-free Mewton-GMRES(m) method with adaptive preconditioner and its application for power flow calculations,"IEEE Trans. Power Syst., vol. 21, no. 3, pp. 1096-1103, Aug.2006.

[30] Y. Tamura, H. Mori and S. Iwamoto "'Relationship Between Voltage Instability and Multiple Load Flow Solutions in Electric Power Systems," IEEE Trans. Power App.Syst., , vol. PAS-102, no. 5. pp.1115 - 1125.May. 1983.

[31] Sasson. A.M, Trevino. C, Aboytes. F "Improved Newton's Load Flow Through a Minimization Technique," IEEE Trans. Power App.Sys., vol. PAS-90.pp. 1974 - 1981, February.1971.

[32] Alves. D.A, da Silva.L.C.P, Castro. C.A and da Costa, V.F," Continuation Fast Decoupled Power Flow With Secant Predictor," IEEE Trans. Power Syst., vol. 16, no.3, pp. 1078 - 1085.August .2003.

[33] Semlyen. A.and de Le'on. F , "Quasi-Newton Power Flow Using Partial Jacobian Updates," IEEE Trans. Power Syst., vol. 16, no.3, pp. 332339.Aug .2001.

[34] Jabr, R.A " A Conic Quadratic Format for the Load Flow Equations of Meshed Networks," IEEE Trans. Power Syst., vol.22, no.4, pp. 2285 2286.Nove .2007

[35] Wang. Y, da Silva. L.C.P and Wilsun Xu," Investigation of the relationship between ill-conditioned power flow and voltage collapse," IEEE Power Engineering Review, vol.20.no.4, pp. 43-45.July .2000.

[36] Yorino, N. Hua-Qiang Li Sasaki, H " A predictor/corrector scheme for obtaining Q-limit points for power flow studies", IEEE Trans. Power Syst., vol.20. pp.130-137.Feb . 2005.

[37] Dimitrovski, A. Tomsovic, K.," Boundary load flow solutions ,"IEEE Trans. Power Syst., vol.19.pp.348-355..Feburary . 2004. 
[38] Sode-Yome. A, Mithulananthan. N and Lee, K.Y. ,"A maximum loading margin method for static voltage stability in power systems ", IEEE Trans. Power Syst., vol.21.pp.799 -808.May. 2004.

[39] R. B. L. Guedes, L. F. C. Alberto and N. G. Bretas, "Power system lowvoltage solutions using an auxiliary gradient system for voltage collapse purposes", IEEE Trans. Power Syst., Vol. 20, No. 3, pp 1528-1537, Aug. 2005.

[40] E.Z. Zhou," Object-oriented Programming, C++ and Power System Simulation”, IEEE Trans. Power Syst., Vol. 11, No. 1, pp.206 -215, Feb .1996. 